\title{
On isometries of the Carathéodory and Kobayashi metrics on strongly pseudoconvex domains
}

\author{
HARISH SESHADRI AND KAUSHAL VERMA
}

\begin{abstract}
Let $\Omega_{1}$ and $\Omega_{2}$ be strongly pseudoconvex domains in $\mathbb{C}^{n}$ and $f$ : $\Omega_{1} \rightarrow \Omega_{2}$ an isometry for the Kobayashi or Carathéodory metrics. Suppose that $f$ extends as a $C^{1}$ map to $\bar{\Omega}_{1}$. We then prove that $\left.f\right|_{\partial \Omega_{1}}: \partial \Omega_{1} \rightarrow \partial \Omega_{2}$ is a CR or anti-CR diffeomorphism. It follows that $\Omega_{1}$ and $\Omega_{2}$ must be biholomorphic or anti-biholomorphic.
\end{abstract}

Mathematics Subject Classification (2000): 32F45 (primary); 32Q45 (secondary).

\section{Introduction}

Complex Finsler metrics such as the Carathéodory and Kobayashi [14] metrics and Kähler metrics such as the Bergman and Cheng-Yau Kähler-Einstein metrics [5] have proved to be very useful in the study functions of several complex variables. Since biholomorphic mappings are isometries for these metrics, they are referred to as 'intrinsic'.

This work is motivated by the question of whether (anti)-biholomorphic mappings are the only isometries for these metrics, i.e., is any isometry $f: \Omega_{1} \rightarrow \Omega_{2}$ between two domains $\Omega_{1}$ and $\Omega_{2}$ in $\mathbb{C}^{n}$ (on which the appropriate intrinsic metrics are non-degenerate) holomorphic or anti-holomorphic?

To be more precise by what we mean by an isometry, let $F_{\Omega}$ and $d_{\Omega}$ denote an intrinsic Finsler metric and the induced distance on a domain $\Omega$. In this paper by a $C^{0}$-isometry we mean a distance-preserving bijection between the metric spaces $\left(\Omega_{1}, d_{\Omega_{1}}\right)$ and $\left(\Omega_{2}, d_{\Omega_{2}}\right)$. For $k \geq 1$, a $C^{k}$-isometry is a $C^{k}$-diffeomorphism $f$ from $\Omega_{1}$ to $\Omega_{2}$ with $f^{*}\left(F_{\Omega_{2}}\right)=F_{\Omega_{1}}$. A $C^{k}$-isometry, $k \geq 1$, is a $C^{0}$-isometry and if the Finsler metric comes from a smooth Riemannian metric (as is the case with the Bergman and the Cheng-Yau metrics), the converse is also true by a classical theorem of Myers and Steenrod.

Both authors were supported by DST (India) Grant No. SR/S4/MS-283/05.

Received December 7, 2005; accepted in revised form July 28, 2006. 
The question above makes sense for a large class of domains (for example bounded domains). However, we confine ourselves to bounded strongly pseudoconvex domains in this paper.

We note that the question has been answered in the affirmative (for strongly pseudoconvex domains) for the Bergman and the Kähler-Einstein metrics in [12]. The proof is essentially based on the fact that the metric under consideration is a Kähler metric whose holomorphic sectional curvatures tend to -1 as one approaches the boundary of the domain. Note that the Bergman metric and the KählerEinstein metric both have this property.

The case of the Carathéodory and the Kobayashi metrics is more delicate. A technical reason is that these metrics are Finsler, not Riemannian, and moreover they are just continuous and not smooth for general strongly pseudoconvex domains. Despite these issues, the results in this paper indicate that the answer to the main question might be in the affirmative.

Before stating our results we remark that all domains we consider have at least $C^{2}$-boundaries. Our main theorem asserts that, an isometry is indeed a holomorphic mapping at 'infinity'.

Theorem 1.1. Let $f: \Omega_{1} \rightarrow \Omega_{2}$ be a $C^{1}$-isometry of two bounded strongly pseudoconvex domains in $\mathbb{C}^{n}$ equipped with the Kobayashi metrics. Suppose that $f$ extends as a $C^{1}$ map to $\bar{\Omega}_{1}$. Then $\left.f\right|_{\partial \Omega_{1}}: \partial \Omega_{1} \rightarrow \partial \Omega_{2}$ is a CR or anti-CR diffeomorphism. Hence $\Omega_{1}$ and $\Omega_{2}$ must be holomorphic or anti-biholomorphic.

A similar statement holds for the inner-Carathéodory metric if we assume that $\partial \Omega_{1}$ and $\partial \Omega_{2}$ are $C^{3}$-smooth.

A few comments about the $C^{1}$-extension assumption: any $C^{1}$-isometry between strongly pseudoconvex domains $\Omega_{1}$ and $\Omega_{2}$ equipped with the Kobayashi or inner-Carathéodory metrics extends to a $C^{1 / 2}$ (Hölder continuous with exponent 1/2) map of $\bar{\Omega}_{1}$ by the results of [1]. See also the remark after Lemma 3.2. A key ingredient in the proof of this result is that strongly pseudoconvex domains equipped with the Kobayashi or inner-Carathéodory metrics are Gromov hyperbolic.

It would be interesting and desirable to prove the $C^{1}$ - extension property for Kobayashi/inner-Carathéodory isometries and hence render the assumption in Theorem 1.1 unnecessary. More precisely, what needs to be investigated is whether $C^{1 / 2}$-boundary regularity implies $C^{1}$-boundary regularity of the given $C^{1}$-isometry. This is known to be valid for holomorphic mappings and the reader is referred to $[19,24]$ and [8] among others for further details. Thus the study of isometries seems to fit in naturally with the study of the boundary behaviour of holomorphic mappings.

We now summarize the ideas behind the proof of Theorem 1.1. The main idea is to use the rescaling technique of Pinchuk [25] to study the derivative of the isometry at a boundary point. We construct a sequence of rescalings of the isometry near a boundary point $p$ and show that this sequence converges (see Proposition 3.2 and 3.3) to an (anti)-holomorphic automorphism of the unbounded realization 
of the ball in $\mathbb{C}^{n}$. On the other hand, we observe that the horizontal components of these rescalings converge to the horizontal component of the derivative of the isometry at $p$. Here by a 'horizontal' vector we mean a vector in the maximal complex subspace of the tangent space at a boundary point of the domain. In fact, we show that the restriction of the derivative to the horizontal subspace at $p$ can be related to the values of the holomorphic automorphism acting on certain points in the ball (see Lemma 4.2). This fact is crucial; indeed, in conjunction with the (anti)-holomorphic limit of the scaled isometries, it allows us to show the complexlinearity of the derivative on the horizontal subspace of the tangent space of $p$. Much of the technical work in the proof is in showing the convergence of metrics under Pinchuk rescalings which is needed to show that the scaled isometries are a 'normal' family. This problem is not faced when dealing with scaled holomorphic mappings.

To the best of our knowledge, this interpretation (cf. Lemma 4.2) of Pinchuk rescaling that relates the derivative on the boundary in the horizontal direction to values of the (anti)-holomorphic limit at certain points in the ball is new. It may be possible to use this interpretation to re-formulate the question about boundary regularity of holomorphic mappings in terms of the scaling method.

A related theme that has been studied in detail is the following: What can be said about a holomorphic mapping between two domains in $\mathbb{C}^{n}$ which is known to be an isometry in some fixed Finsler metric in the domain and range, at a single point? The reader is referred to [4, 11, 20] and [29] which study this question, the conclusion in all being that the holomorphic mapping is biholomorphic. The main theorem dispenses with the hypothesis of having a global holomorphic mapping and replaces it with a global isometry at the expense of assuming some apriori boundary regularity.

The first important technical lemma that we need is about the behaviour of the distance to the boundary under isometries (Lemma 2.2). Here we use the two-sided estimates for the Kobayashi distance obtained in [1].

\section{Preliminaries}

\subsection{The Kobayashi and Carathéodory metrics}

Let $\Delta$ denote the open unit disc in $\mathbb{C}$ and let $\rho(a, b)$ denote the distance between two points $a, b \in \Delta$ with respect to the Poincáre metric (of constant curvature -4).

Let $\Omega$ be a domain in $\mathbb{C}^{n}$. The Kobayashi, the Carathéodory and the innerCarathéodory distances on $\Omega$, denoted by $d_{\Omega}^{K}, d_{\Omega}^{C}$ and $d_{\Omega}^{\tilde{C}}$ respectively, are defined as follows:

Let $z \in \Omega$ and $v \in T_{z} \Omega$ a tangent vector at $z$. Define the associated infinitesimal Kobayashi and Carathéodory metrics as

$$
F_{\Omega}^{K}(z, v)=\inf \left\{\frac{1}{\alpha}: \alpha>0, \phi \in \mathcal{O}(\Delta, \Omega) \text { with } \phi(0)=z, \phi^{\prime}(0)=\alpha v\right\}
$$


and

$$
F_{\Omega}^{\tilde{C}}(z, v)=\sup \{|d f(z) v|: f \in \mathcal{O}(\Omega, \Delta)\},
$$

respectively. The inner-Carathéodory length and the Kobayashi length of a piecewise $C^{1}$ curve $\sigma:[0,1] \rightarrow \Omega$ are given by

$$
l^{\tilde{C}}(\sigma)=\int_{0}^{1} F_{\Omega}^{\tilde{C}}\left(\sigma, \sigma^{\prime}\right) \mathrm{d} t \text { and } l^{K}(\sigma)=\int_{0}^{1} F_{\Omega}^{K}\left(\sigma, \sigma^{\prime}\right) \mathrm{d} t,
$$

respectively. Finally the Kobayashi and inner-Carathéodory distances between $p, q$ are defined by

$$
d_{\Omega}^{K}(p, q)=\inf \left\{l^{K}(p, q)\right\} \text { and } d_{\Omega}^{\tilde{C}}(p, q)=\inf \left\{l^{\tilde{C}}(p, q)\right\},
$$

where the infimums are taken over all piece-wise $C^{1}$ curves in $\Omega$ joining $p$ and $q$.

The Carathéodory distance is defined to be

$$
d_{\Omega}^{C}(p, q)=\sup \{\rho(f(p), f(q)): f \in \mathcal{O}(\Omega, \Delta)\} .
$$

We note the following well-known and easy facts:

- If $\Omega$ is a bounded domain, then $d_{\Omega}^{K}, d_{\Omega}^{C}$ and $d_{\Omega}^{\tilde{C}}$ are non-degenerate and the topology induced by these distances is the Euclidean topology.

- These distance functions are invariant under biholomorphisms. More generally, holomorphic mappings are distance non-increasing. The same holds for $F_{\Omega}^{K}(z, v)$ and $F_{\Omega}^{\tilde{C}}(z, v)$.

- We always have

$$
d_{\Omega}^{C}(p, q) \leq d_{\Omega}^{\tilde{C}}(p, q) \leq d_{\Omega}^{K}(p, q) .
$$

- If $\Omega=\mathbb{B}^{n}$, all the distance functions above coincide and are equal to the distance function of the Bergman metric $g_{0}$ on $\mathbb{B}^{n}$. Here the Bergman metric is a complete Kähler metric normalized to have constant holomorphic sectional curvature -4 . Also, for $\mathbb{B}^{n}$, the infinitesimal Kobayashi and Carathéodory metrics are both equal to the quadratic form associated to $g_{0}$.

\subsection{Convexity and Pseudoconvexity}

Suppose $\Omega$ is a bounded domain in $\mathbb{C}^{n}, n \geq 2$, with $C^{2}$-smooth boundary. Let $\rho: \mathbb{C}^{n} \rightarrow \mathbb{R}$ be a smooth defining function for $\Omega$, i.e, $\rho=0$ on $\partial \Omega, d \rho \neq 0$ at any point of $\partial \Omega$ and $\rho^{-1}(-\infty, 0)=\Omega$.

A domain with $C^{2}$ smooth boundary $\Omega$ is said to be strongly convex if there is a defining function $\rho$ for $\partial \Omega$ such that the real Hessian of $\rho$ is positive definite as a bilinear form on $T_{p} \partial \Omega$, for every $p \in \partial \Omega$.

$\Omega$ is strictly convex if the interior of the straight line segment joining any two points in $\bar{\Omega}$ is contained in $\Omega$. Note that we do not demand the boundary of $\Omega$ be smooth. Strong convexity implies strict convexity. 
Let $\Omega$ be a bounded domain. A holomorphic map $\phi: \Delta \rightarrow \Omega$ is said to be an extremal disc or a complex geodesic for the Kobayashi metric (or distance) if it is distance preserving, i.e. $d_{\Omega}^{K}(\phi(p), \phi(q))=\rho(p, q)$ for all $p, q \in \Delta$.

The following fundamental theorem about complex geodesics in strictly convex domains will be repeatedly used in Section 3 of this paper:

Theorem 2.1 (L. Lempert [16]). Let $\Omega$ be a bounded strictly convex domain in $\mathbb{C}^{n}$.

(1) Given $p \in \Omega$ and $v \in \mathbb{C}^{n}$, there exists a complex geodesic $\phi$ with $\phi(0)=p$ and $\phi^{\prime}(0)=v\left(\right.$ or $d \phi\left(T_{0} \Delta\right)=P_{v}$, where $P_{v} \subset T_{p} \Omega$ is the real -two plane generated by the complex vector $v)$.

$\phi$ also preserves the infinitesimal metric, i.e., $F_{\Omega}^{K}(\phi(q) ; d \phi(w))=F_{\Delta}(q ; w)$ for all $w \in T_{q} \Delta$.

(2) Given $p$ and $q$ in $\Omega$, there exists a complex geodesic $\phi$ whose image contains $p$ and $q$.

(3) The Kobayashi, Carathéodory and inner-Carathéodory distances coincide on $\Omega$. Also, the Kobayashi and Carthéodory infinitesimal metrics coincide on $\Omega$.

The Levi form of the defining function $\rho$ at $p \in \mathbb{C}^{n}$ is defined by

$$
L_{p}(v)=\sum_{i, j=1}^{n} \frac{\partial^{2} \rho}{\partial z_{i} \partial \bar{z}_{j}}(p) v_{i} \bar{v}_{j} \text { for } v=\left(v_{1}, . ., v_{n}\right) \in \mathbb{C}^{n}
$$

For $p \in \partial \Omega$, the maximal complex subspace of the tangent space $T_{p} \partial \Omega$ is denoted by $H_{p}(\partial \Omega)$ and called the horizontal subspace at $p$. By definition, $\Omega$ is strongly pseudoconvex if $L_{p}$ is positive definite on $H_{p}(\partial \Omega)$ for all $p \in \partial \Omega$. It can be checked that strong convexity implies strong pseudoconvexity.

For a strongly pseudoconvex domain, the Carnot-Carathéodory metric on $\partial \Omega$ is defined as follows. A piecewise $C^{1}$ curve $\alpha:[0,1] \rightarrow \partial \Omega$ is called horizontal if $\dot{\alpha}(t) \in H_{\alpha(t)}(\partial \Omega)$ wherever $\dot{\alpha}(t)$ exists. The strong pseudoconvexity of $\Omega$ implies that $\partial \Omega$ is connected and, in fact, any two points can be connected by a horizontal curve. The Levi-length of a curve $\alpha$ is defined by $l(\alpha)=\int_{0}^{1} L_{\alpha(t)}(\dot{\alpha}(t))^{\frac{1}{2}} \mathrm{~d} t$. Finally the Carnot-Carathéodory metric is defined, for any $p, q \in \partial \Omega$, by

$$
d_{H}(p, q)=\inf _{\alpha} l(\alpha)
$$

where the infimum is taken over horizontal curves $\alpha:[0,1] \rightarrow \partial \Omega$ with $\alpha(0)=p$ and $\alpha(1)=q$.

\subsection{Notation}

$\circ \Delta:=\{z \in \mathbb{C}:|z|<1\}, \Delta_{r}:=\{z \in \mathbb{C}:|z|<r\}$.

$\circ \rho=$ distance function on $\Delta$ of the Poincáre metric of curvature -4 . 
○ For $n \geq 2, \mathbb{B}^{n}:=\left\{z \in \mathbb{C}^{n}:|z|<1\right\}$ and $B_{a}(r)=\left\{z \in \mathbb{C}^{n}:|z-a|<r\right\}$.

$\circ \Sigma_{z}=\left\{z=\left(z_{1}, \ldots, z_{n}\right) \in \mathbb{C}^{n}: 2 \operatorname{Re} z_{n}+\left|z_{1}\right|^{2}+\ldots+\left|z_{n-1}\right|^{2}<0\right\}$, the unbounded realization of the ball $\mathbb{B}^{n}$, which is a Siegel domain.

○ $H_{p}(\partial \Omega) \subset T_{p} \partial \Omega$ denotes the horizontal subspace of $T_{p} \partial \Omega, p \in \partial \Omega$.

○ Given $p \in \partial \Omega$, for any $v \in \mathbb{C}^{n}, v=v_{H}+v_{N}$ corresponds to $\mathbb{C}^{n}=H_{p}(\partial \Omega) \oplus$ $H_{p}(\partial \Omega)^{\perp}$.

○ $z=\left(\tilde{z}, z_{n}\right)$ corresponds to $\mathbb{C}^{n}=\mathbb{C}^{n-1} \times \mathbb{C}$.

$\circ \delta(x)=d(x, \partial \Omega)$ denotes Euclidean distance of $x \in \Omega$ to $\partial \Omega$.

$\circ d_{\Omega}^{K}, d_{\Omega}^{C}$ and $d_{\Omega}^{\tilde{C}}$ denote the Kobayashi, Carathéodory and inner-Carathéodory distances on $\Omega$.

- $F_{\Omega}^{K}$ and $F_{\Omega}^{C}$ denote the Kobayashi and Carathéodory infinitesimal metrics on $\Omega$.

- If $f: \Omega_{1} \rightarrow \Omega_{2}$ is a smooth map between domains $\Omega_{1}$ and $\Omega_{2}$ in $\mathbb{C}^{n}$, then $d f_{p}: \mathbb{R}^{2 n} \rightarrow \mathbb{R}^{2 n}$ denotes the derivative at $p \in \Omega_{1}$.

Finally, the letters $C$ or $c$ will be used to denote an arbitrary constant throughout this article and which is subject to change, even within the limits of a given line, unless otherwise stated.

\subsection{An estimate for the distance to the boundary}

We prove that $C^{0}$-isometries approximately preserve the distance to the boundary. This is needed for the convergence of Pinchuk rescalings in Section 3. For a domain $\Omega$ and a point $x \in \Omega, \delta(x)$ denotes the Euclidean distance $\delta(x)=d(x, \partial \Omega)$. Our proof uses the results and notations of [1] in a crucial way and we refer the reader to it for further details.

We note that in the lemma below we do not need to assume that the isometry has a $C^{1}$-extension to the closure of the domain.

Lemma 2.2. Let $\Omega_{1}$ and $\Omega_{2}$ be strongly pseudoconvex domains in $\mathbb{C}^{n}$ and $f$ : $\Omega_{1} \rightarrow \Omega_{2}$ a $C^{0}$ isometry of the Kobayashi on $\Omega_{1}$ and $\Omega_{2}$. There exist positive constants $A$ and $B$ such that

$$
B \delta(x) \leq \delta(f(x)) \leq A \delta(x)
$$

for all $x \in \Omega_{1}$. A similar statement holds for an isometry of the inner-Carathéodory distance if we assume that $\partial \Omega_{1}$ and $\partial \Omega_{2}$ are $C^{3}$ smooth.

Proof. Since $\Omega$ has a $C^{2}$ boundary, given $x \in \Omega$ sufficiently close to the boundary, there exists a unique point $\pi(x) \in \partial \Omega$ such that $|x-\pi(x)|=\delta(x)$. Extend the domain of $\pi$ to be all of $\Omega$. Such an extension is not uniquely defined but any extension will work for our purposes. 
Following [1], define for any strongly pseudoconvex $\Omega$, the function $g: \Omega \times \Omega \rightarrow$ $\mathbb{R}$ by

$$
g(x, y)=2 \log \left[\frac{d_{H}(\pi(x), \pi(y))+\max \{h(x), h(y)\}}{\sqrt{h(x) h(y)}}\right],
$$

where $h(x)=\sqrt{\delta(x)}$ and $d_{H}$ is the Carnot-Carathéodory metric on $\partial \Omega$.

The Box-Ball estimate (Proposition 3.1 of [1]) implies that the topology induced by $d_{H}$ on $\partial \Omega$ agrees with the Euclidean topology. Hence, $\left(\partial \Omega, d_{H}\right)$ is compact and, in particular, has finite diameter, say $D$. This implies that

$$
2 \log \sqrt{\frac{h(y)}{h(x)}} \leq g(x, y) \leq 2 \log \left(\frac{D+S}{\sqrt{h(x) h(y)}}\right)
$$

where we have used $\max \{h(x), h(y)\} \geq h(y)$ in the first inequality and where $S=\sup _{x \in \Omega} h(x)$. Hence

$$
\frac{h(y)}{h(x)} \leq e^{g(x, y)} \leq \frac{E}{h(x) h(y)} .
$$

Now we consider the functions $g_{1}$ and $g_{2}$ associated to $\Omega_{1}$ and $\Omega_{2}$. By Corollary 1.3 of [1], there exists a constant $C_{1}$ such that

$$
g_{1}(x, y)-C_{1} \leq d_{\Omega_{1}}^{K}(x, y) \leq g_{1}(x, y)+C_{1}
$$

for all $x, y$ in $\Omega_{1}$.

According to [2], such an estimate holds for the inner-Carathéodory distance as well, if one assumes $C^{3}$-regularity of the boundaries.

Combining (2.2) and (2.1) gives

$$
A_{1} \frac{h(y)}{h(x)} \leq e^{d_{\Omega_{1}}^{K}(x, y)} \leq \frac{B_{1}}{h(x) h(y)} .
$$

A similar inequality holds on $\Omega_{2}$ (with $A_{2}$, etc). Fixing $y \in \Omega_{1}$, using $d_{\Omega_{1}}^{K}(x, y)=$ $d_{\Omega_{2}}^{K}(f(x), f(y))$, and comparing the inequalities on $\Omega_{1}$ and $\Omega_{2}$, we get the required estimates. The proof for the inner-Carathéodory distance is the same.

An immediate corollary of Lemma 2.2 is that for $C^{1}$-isometries which have $C^{1}$ extensions, the derivative of the boundary map preserves the horizontal distribution of $T$. Note that necessarily $f\left(\partial \Omega_{1}\right) \subset \partial \Omega_{2}$, by Lemma 2.2 .

Lemma 2.3. Let $f: \Omega_{1} \rightarrow \Omega_{2}$ be a $C^{1}$-isometry of strongly pseudoconvex domains equipped with the Kobayashi metric. If $f$ extends to a $C^{1}$-map of $\bar{\Omega}_{1}$, then

$$
d f_{p}\left(H_{p}\left(\partial \Omega_{1}\right)\right) \subset H_{f(p)}\left(\partial \Omega_{2}\right)
$$

for any $p \in \partial \Omega$. This holds for an isometry of the inner-Carathéodory metric as well if we assume that $\partial \Omega_{1}$ and $\partial \Omega_{2}$ are $C^{3}$-smooth. 
Proof. By [17], there exists $\delta_{0}>0$ such that for any $x \in \Omega_{1}$ with $\delta(x) \leq \delta_{0}$ and for all $v=v_{H}+v_{N} \in \mathbb{C}^{n}$ (where this decomposition is taken at $\pi(x)$ ), we have

$$
\frac{\left|v_{N}\right|^{2}}{8 \delta(x)^{2}}+\frac{L_{\pi(x)}\left(v_{H}\right)}{4 \delta(x)} \leq\left(F_{\Omega_{1}}^{K}(x, v)\right)^{2} \leq \frac{\left|v_{N}\right|^{2}}{2 \delta(x)^{2}}+4 \frac{L_{\pi(x)}\left(v_{H}\right)}{\delta(x)} .
$$

One has similar estimates for $d f_{x}(v)=d f_{x}(v)_{H}+d f_{x}(v)_{N}$. Since $f$ is an isometry we have $F_{\Omega_{1}}^{K}(x, v)=F_{\Omega_{2}}^{K}\left(f(x), d f_{x}(v)\right)$. Now assume that $v \in H_{p}\left(\partial \Omega_{1}\right)$, i.e., $v=v_{H}$. Comparing the estimates (corresponding to (2.3)) for $v$ and $d f_{x}(v)$, we get

$$
\frac{\left|d f_{x}(v)_{N}\right|^{2}}{8 \delta(f(x))^{2}}+\frac{L_{\pi(f(x))}\left(d f_{x}(v)_{H}\right)}{4 \delta(f(x))} \leq 4 \frac{L_{\pi(x)}\left(v_{H}\right)}{\delta(x)} .
$$

We can assume that $L_{\pi(x)}(w) \leq c|w|^{2}$ for all $w \in H_{q}\left(\partial \Omega_{1}\right), q \in \partial \Omega_{1}$. Combining this with Lemma 2.2 and (2.4), we get

$$
\left|d f_{x}(v)_{N}\right| \leq C \delta(x)|v|
$$

for some uniform positive constant $C$. Letting $x \rightarrow p$ and using the continuity of $d f$ we obtain $d f_{p}(v)_{N}=0$.

Remark: Once it known that $\delta(x) \approx \delta(f(x))$ uniformly for all $x \in \Omega_{1}$, it follows by well known arguments that $f \in C^{1 / 2}\left(\bar{\Omega}_{1}\right)$. Indeed, from (2.3) and the fact that $f$ is an isometry we get

$$
C \frac{\left|d f_{x}(v)\right|}{\sqrt{\delta(f(x))}} \leq F_{\Omega_{2}}^{K}\left(f(x), d f_{x}(v)\right)=F_{\Omega_{1}}^{K}(x, v) \leq \frac{|v|}{\delta(x)}
$$

for all tangent vectors $v$ at $x$. Hence

$$
\left|d f_{x}(v)\right| \leq C \frac{|v|}{\sqrt{\delta(x)}}
$$

Integrating this along a polygonal path as in [22] yields

$$
|f(p)-f(q)| \leq C|p-q|^{1 / 2}
$$

uniformly for all $p, q$ in $\Omega_{1}$.

\section{A metric version of Pinchuk rescaling}

Throughout this section, we will assume that the boundary of the domain under consideration is $C^{3}$-smooth when dealing with the inner-Carthéodory distance. Otherwise we assume that $\partial \Omega$ is $C^{2}$. 
Let $p \in \partial \Omega$, and fix a sequence $\left\{p_{n}\right\}$ in $\Omega$ converging to $p$. It has been shown in Lemma 2.2 that

$$
d\left(p_{n}, \partial \Omega_{1}\right) \approx d\left(f\left(p_{n}\right), \partial \Omega_{2}\right) .
$$

In particular $\left\{f\left(p_{n}\right)\right\}$ will cluster only on $\partial \Omega_{2}$. By passing to a subsequence we can assume that $q_{n}=f\left(p_{n}\right) \rightarrow q \in \partial \Omega_{2}$. Fix a defining function $\rho$ for $\partial \Omega_{1}$ that is strongly plurisubharmonic and of class $C^{2}$ in some neighbourhood of $\bar{\Omega}_{1}$. Similarly let $\rho^{\prime}$ be such a function for $\Omega_{2}$. The following lemma in [23] will be vital for what follows.

Lemma 3.1. Let $\Omega$ be a strongly pseudoconvex domain, $\rho$ a defining function for $\partial \Omega$, and $p \in \partial \Omega$. Then there exists a neighbourhood $U$ of $p$ and a family of biholomorphic mappings $h_{\zeta}: \mathbb{C}^{n} \rightarrow \mathbb{C}^{n}$ depending continuously on $\zeta \in \partial \Omega \cap U$ that satisfy the following:

(i) $h_{\zeta}(\zeta)=0$.

(ii) The defining function $\rho_{\zeta}=\rho \circ h_{\zeta}^{-1}$ of the domain $\Omega^{\zeta}:=h_{\zeta}(\Omega)$ has the form

$$
\rho_{\zeta}(z)=2 \operatorname{Re}\left(z_{n}+K_{\zeta}(z)\right)+H_{\zeta}(z)+\alpha_{\zeta}(z)
$$

where $K_{\zeta}(z)=\sum_{i, j=1}^{n} a_{i j}(\zeta) z_{i} z_{j}, H_{\zeta}(z)=\sum_{i, j=1}^{n} a_{i} \bar{j}(\zeta) z_{i} \bar{z}_{j}$ and $\alpha_{\zeta}(z)=$ $o\left(|z|^{2}\right)$ with $K_{\zeta}(\tilde{z}, 0) \equiv 0$ and $H_{\zeta}(\tilde{z}, 0) \equiv|\tilde{z}|^{2}$.

(iii) The mapping $h_{\zeta}$ takes the real normal to $\partial \Omega$ at $\zeta$ to the real normal $\left\{\tilde{z}=y_{n}=\right.$ $0\}$ to $\partial \Omega^{\zeta}$ at the origin.

To apply this lemma select $\zeta_{k} \in \partial \Omega_{1}$, closest to $p_{k}$ and $w_{k} \in \partial \Omega_{2}$ closest to $q_{k}=f\left(p_{k}\right)$. For $k$ large, the choice of $\zeta_{k}$ and $w_{k}$ is unique since $\partial \Omega_{1}$ and $\partial \Omega_{2}$ are sufficiently smooth. Moreover $\zeta_{k} \rightarrow p$ and $w_{k} \rightarrow q$. Let $h_{k}:=h_{\zeta_{k}}$ and $g_{k}:=g_{w_{k}}$ be the biholomorphic mappings provided by the lemma above. Let

$$
\Omega_{1}^{k}:=h_{k}\left(\Omega_{1}\right), \quad \Omega_{2}^{k}:=g_{k}\left(\Omega_{2}\right) \text { and } f_{k}:=g_{k} \circ f \circ h_{k}^{-1}: \Omega_{1}^{k} \rightarrow \Omega_{2}^{k} .
$$

Note that $f_{k}$ is also an isometry for the Kobayashi distance on $\Omega_{1}^{k}$ and $\Omega_{2}^{k}$.

Let $T_{k}: \mathbb{C}^{n} \rightarrow \mathbb{C}^{n}$ be the anisotropic dilatation map given by

$$
T_{k}\left(\tilde{z}, z_{n}\right)=\left(\frac{1}{\sqrt{\delta}_{k}} \tilde{z}, \frac{1}{\delta_{k}} z_{n}\right)
$$

and let

$$
\tilde{\Omega}_{1}^{k}:=T_{k}\left(\Omega_{1}^{k}\right), \quad \tilde{\Omega}_{2}^{k}:=T_{k}\left(\Omega_{2}^{k}\right) \text { and } \Phi_{k}:=T_{k} \circ f_{k} \circ T_{k}^{-1}: \tilde{\Omega}_{1}^{k} \rightarrow \tilde{\Omega}_{2}^{k} .
$$

Again $\Phi_{k}$ is an isometry. Let us note that the explicit expression for $\Phi_{k}$ is

$$
\Phi_{k}(z)=\left(\frac{1}{\sqrt{\delta}_{k}} \tilde{f}_{k}\left(\sqrt{\delta}_{k} \tilde{z}, \delta_{k} z_{n}\right), \frac{1}{\delta_{k}} \hat{f}_{k}\left(\sqrt{\delta}_{k} \tilde{z}, \delta_{k} z_{n}\right)\right) .
$$


For notational convenience, let us denote the compositions of the rotations and the scalings by

$$
H_{k}:=T_{k} \circ h_{k} \text { and } I_{k}:=T_{k} \circ g_{k} \Rightarrow \Phi_{k}=I_{k} \circ f \circ H_{k} .
$$

Note that the defining functions for $\tilde{\Omega}_{1}^{k}$ and $\tilde{\Omega}_{2}^{k}$ are given by

$$
\tilde{\rho}_{k}(z)=\frac{1}{\sqrt{\delta}_{k}} \rho_{k}\left(\sqrt{\delta}_{k} \tilde{z}, \delta_{k} z_{n}\right), \quad \tilde{\rho}_{k}^{\prime}(w)=\frac{1}{\sqrt{\delta}_{k}} \rho_{k}\left(\sqrt{\delta}_{k} \tilde{w}, \delta_{k} w_{n}\right)
$$

respectively.

The family of functions $\left\{h_{k}\right\}$ converges uniformly on compact subsets of $\mathbb{C}^{n}$ to the identity mapping, as do their inverses $h_{k}^{-1}$. Thus it follows that for $k \gg 1$

$$
\frac{1}{B} \leq \frac{d\left(h_{k}(z), \partial \Omega_{1}^{k}\right)}{d\left(z, \partial \Omega_{1}\right)} \leq B \text { and } \frac{1}{B} \leq \frac{d\left(g_{k}(w), \partial \Omega_{2}^{k}\right)}{d\left(w, \partial \Omega_{2}\right)} \leq B
$$

for some constant $B$ independent of $z$ and $k$.

Combining (3.1) and (3.3) shows that for $k \gg 1$

$$
\frac{1}{c} d\left(z, \partial \Omega_{1}^{k}\right) \leq d\left(f_{k}(z), \partial \Omega_{2}^{k}\right) \leq c d\left(z, \partial \Omega_{1}^{k}\right)
$$

where $c$ is independent of $k$, for $k \gg 1$ and $z \in \Omega_{1}^{k}$. Moreover, since $\rho$ and $\rho^{\prime}$ (and hence $\rho_{k}:=\rho_{\zeta_{k}}=\rho \circ h_{k}^{-1}$ and $\rho_{k}^{\prime}:=\rho_{w_{k}}^{\prime}=\rho^{\prime} \circ g_{k}^{-1}$ ) are smooth, it follows that there exists a uniform constant $c>0$ such that

$$
\frac{1}{c} \leq \frac{d\left(z, \partial \Omega_{1}^{k}\right)}{\left|\rho_{k}(z)\right|} \leq c \text { and } \frac{1}{c} \leq \frac{d\left(w, \partial \Omega_{2}^{k}\right)}{\left|\rho_{k}^{\prime}(w)\right|} \leq c
$$

for $k \gg 1$ and $z \in \Omega_{1}^{k}, w \in \Omega_{2}^{k}$. Let $\delta_{k}=d\left(h_{k}\left(p_{k}\right), \partial \Omega_{1}^{k}\right)$ and $\gamma_{k}=d\left(g_{k}\left(q_{k}\right), \partial \Omega_{2}^{k}\right)$.

Two observations can be made at this stage: first, for $k \gg 1, h_{k}\left(p_{k}\right)=$ $\left(0,-\delta_{k}\right), g_{k}\left(q_{k}\right)=\left(0,-\gamma_{k}\right)$ and $f_{k}\left(0,-\delta_{k}\right)=\left(0,-\gamma_{k}\right)$ as follows from Lemma 3.1, and secondly (3.4) shows that

$$
\frac{1}{c} \delta_{k} \leq \gamma_{k} \leq c \delta_{k}
$$

for some $c>0$.

It has been shown in [23] that the sequence of domains $\left\{\tilde{\Omega}_{1}^{k}\right\}$ converges to the unbounded realization of the unit ball, namely to

$$
\Sigma_{z}=\left\{z \in \mathbb{C}^{n}: 2 \operatorname{Re} z_{n}+|\tilde{z}|^{2}<0\right\} .
$$

The convergence is in the sense of Hausdorff convergence of sets. Similarly $\left\{\tilde{\Omega}_{2}^{k}\right\}$ will converge to $\Sigma_{w}$, the unbounded realization of the ball in $w$ coordinates. 
Proposition 3.2. Let $\Omega_{1}, \Omega_{2}$ be smoothly bounded strongly pseudoconvex domains in $\mathbb{C}^{n}$. Suppose that $f: \Omega_{1} \rightarrow \Omega_{2}$ is a $C^{0}$-isometry with respect to the Kobayashi (respectively inner-Carathéodory) distances on $\Omega_{1}$ and $\Omega_{2}$. Define the sequence of domains $\tilde{\Omega}_{1}^{k}$, $\tilde{\Omega}_{2}^{k}$ and mappings $\Phi_{k}: \tilde{\Omega}_{1}^{k} \rightarrow \tilde{\Omega}_{2}^{k}$. Then there exists a subsequence of $\left\{\Phi_{k}\right\}$ that converges uniformly on compact subsets of $\Sigma_{z}$ to a continuous mapping $\Phi: \Sigma_{z} \rightarrow \mathbb{C}^{n}$.

Proof. The case when $\Phi$ is an isometry with respect to the Kobayashi distance will be dealt with first. By construction $\Phi_{k}(0,-1)=\left(0,-\gamma_{k} / \delta_{k}\right)$ and (3.6) shows that $\left\{\Phi_{k}(0,-1)\right\}$ is bounded. The domain $\Sigma_{z}$ can be exhausted by an increasing union $\left\{S_{i}\right\}$ of relatively compact convex domains each containing $(0,-1)$. Fix a pair $S_{i_{0}} \Subset S_{i_{0}+1}$ and write $S_{1}=S_{i_{0}}$ and $S_{2}=S_{i_{0}+1}$ for brevity. Since $\tilde{\Omega}_{1}^{k}$ converges to $\Sigma_{z}$ it follows that $S_{1} \Subset S_{2} \Subset \tilde{\Omega}_{1}^{k}$ for all $k \gg 1$. It will suffice to show that $\left\{\Phi_{k}\right\}$ restricted to $S_{1}$ is uniformly bounded and equicontinuous. Fix $s_{1}, s_{2}$ in $S_{1}$. The following inequalities hold for large $k$ :

$$
d_{\tilde{\Omega}_{2}^{k}}^{K}\left(\Phi_{k}\left(s_{1}\right), \Phi_{k}\left(s_{2}\right)\right)=d_{\tilde{\Omega}_{1}^{k}}^{K}\left(s_{1}, s_{2}\right) \leq d_{S_{2}}^{K}\left(s_{1}, s_{2}\right) \leq c\left|s_{1}-s_{2}\right|
$$

for $c>0$ independent of $k$. Indeed the equality holds for all $k$ since $\Phi_{k}$ is an isometry and the inequalities are a result of the following observations: first, the inclusion $S_{2} \hookrightarrow \tilde{\Omega}_{1}^{k}$ is distance decreasing for the Kobayashi distance and second, since $S_{2}$ is convex, the infinitesimal Kobayashi metric $F_{S_{2}}^{K}(z, v)$ satisfies

$$
F_{S_{2}}^{K}(z, v) \leq \frac{|v|}{\delta_{v}(z)}
$$

where $z \in S_{2}, v$ is a tangent vector at $z$ and $\delta_{v}(z)$ is the distance of $z$ to $\partial S_{2}$ in the direction along $v$. Now joining $s_{1}$ and $s_{2}$ by a straight line path $\gamma(t)$ and integrating (3.8) along $\gamma(t)$ yields the last inequality in (3.7).

To estimate $d_{\tilde{\Omega}_{2}^{k}}^{K}\left(\Phi_{k}\left(s_{1}\right), \Phi_{k}\left(s_{2}\right)\right)$ note from (3.2) that

$$
d_{\tilde{\Omega}_{2}^{k}}^{K}\left(\Phi_{k}\left(s_{1}\right), \Phi_{k}\left(s_{2}\right)\right)=d_{\Omega_{2}}^{K}\left(f \circ H_{k}^{-1}\left(s_{1}\right), f \circ H_{k}^{-1}\left(s_{2}\right)\right)
$$

since $I_{k}$ is an isometry. Since $f$ is continuous at $p \in \partial \Omega_{1}$, choose neighborhoods $U_{1}, U_{2}$ of $p, q=f(p)$ respectively so that $f\left(U_{1} \cap \Omega_{1}\right) \subset U_{2} \cap \Omega_{2}$. Note that $p_{n}$, and $\zeta_{n}$ as chosen earlier lie in $U_{1} \cap \Omega_{1}$ eventually. For $k$ large, $H_{k}^{-1}\left(S_{1}\right) \subset U_{1} \cap \Omega_{1}$ and hence both $f \circ H_{k}^{-1}\left(s_{1}\right)$ and $f \circ H_{k}^{-1}\left(s_{2}\right)$ lie in $U_{2} \cap \Omega_{2}$. It is well known that the Kobayashi distance can be localized near strongly pseudoconvex points in the sense that for every choice of $U_{2}$, there is a smaller neighborhood $p \in U_{3}, U_{3}$ relatively compact in $U_{2}$, and $c>0$ such that

$$
c d_{U_{2} \cap \Omega_{2}}^{K}(x, y) \leq d_{\Omega_{2}}^{K}(x, y)
$$

for all $x, y \in U_{3} \cap \Omega_{2}$. We apply this to $x=f \circ H_{k}^{-1}\left(s_{1}\right)$ and $y=f \circ H_{k}^{-1}\left(s_{2}\right)$, both of which belong to $U_{3} \cap \Omega_{2}$ for large $k$, by shrinking $U_{1}$ if necessary. 
Moreover, thanks to the strong pseudoconvexity of $\partial \Omega_{2}$ near $q$, it is possible to choose $U_{2}$ small enough so that for $k \gg 1$,

$$
g_{k}\left(U_{2} \cap \Omega_{2}\right) \subset\left\{w \in \mathbb{C}^{n}:\left|w_{n}+R\right|^{2}+|\tilde{w}|^{2}<R^{2}\right\} \subset \tilde{\Omega}
$$

where $\Omega:=\left\{w \in \mathbb{C}^{n}: 2 R\left(\operatorname{Re} w_{n}\right)<-|\tilde{w}|^{2}\right\}$ for some fixed $R>1$.

Note that $\tilde{\Omega}$ is invariant under the dilatation $T_{k}$ for all $k$ and moreover $\tilde{\Omega}$ is biholomorphic to $\mathbb{B}^{n}$. Thus, $T_{k} \circ g_{k}\left(U_{2} \cap \Omega_{2}\right) \subset \tilde{\Omega}$ and hence $\Phi_{k}\left(s_{1}\right), \Phi_{k}\left(s_{2}\right)$ both lie in $\tilde{\Omega}$ for $k$ large. From (3.9) and (3.10) it follows that

$$
c d_{\tilde{\Omega}}^{K}\left(\Phi_{k}\left(s_{1}\right), \Phi_{k}\left(s_{2}\right)\right) \leq d_{\tilde{\Omega}_{2}^{k}}^{K}\left(\Phi_{k}\left(s_{1}\right), \Phi_{k}\left(s_{2}\right)\right)
$$

for $k$ large. Combining (3.7) and (3.11) gives

$$
d_{\tilde{\Omega}}^{K}\left(\Phi_{k}\left(s_{1}\right), \Phi_{k}\left(s_{2}\right)\right) \leq c\left|s_{1}-s_{2}\right|
$$

for $s_{1}, s_{2} \in S_{1}$ and $k \gg 1$.

Let $\psi: \tilde{\Omega} \rightarrow \mathbb{B}^{n}$ be a biholomorphic mapping. To show that $\left\{\Phi_{k}\left(S_{1}\right)\right\}$ is uniformly bounded, choose $s_{1} \in S_{1}$ arbitrarily and $s_{2}=(0,-1)$. Then (3.11) shows that

$$
d_{\tilde{\Omega}}^{K}\left(\Phi_{k}\left(s_{1}\right), \Phi_{k}(0,-1)\right) \leq c\left|s_{1}-s_{2}\right|<\infty .
$$

Since $\left\{\Phi_{k}(0,-1)\right\}$ is bounded and $\mathbb{B}^{n}$ (and hence $\tilde{\Omega}$ ) is complete in the Kobayashi distance, it follows that $\left\{\Phi_{k}\left(s_{1}\right)\right\}$ is bounded.

To show that $\left\{\Phi_{k}\right\}$ restricted to $S_{1}$ is equicontinuous observe that the Kobayashi distance in $\mathbb{B}^{n}$ between $\psi \circ \Phi_{k}\left(s_{1}\right)$ and $\psi \circ \Phi_{k}\left(s_{2}\right)$ equals

$$
d_{\tilde{\Omega}}^{K}\left(\Phi_{k}\left(s_{1}\right), \Phi_{k}\left(s_{2}\right)\right) \leq c\left|s_{1}-s_{2}\right| .
$$

Using the explicit formula for the Kobayashi distance between two points in $\mathbb{B}^{n}$, this gives

$$
\left|\frac{\psi \circ \Phi_{k}\left(s_{1}\right)-\psi \circ \Phi_{k}\left(s_{2}\right)}{1-\overline{\psi \circ \Phi_{k}\left(s_{1}\right)} \psi \circ \Phi_{k}\left(s_{2}\right)}\right| \leq \frac{\exp \left(2 c\left|s_{1}-s_{2}\right|\right)-1}{\exp \left(2 c\left|s_{1}-s_{2}\right|\right)+1}
$$

Since $\left\{\Phi_{k}\left(S_{1}\right)\right\}$ is relatively compact in $\tilde{\Omega}$ for $k \gg 1$, it follows that so is $\{\psi \circ$ $\left.\Phi_{k}\left(S_{1}\right)\right\}$ in $\mathbb{B}^{n}$. Let $\left\{\psi \circ \Phi_{k}\left(S_{1}\right)\right\} \subset G \Subset \mathbb{B}^{n}$. Hence

$$
\left|1-\overline{\psi \circ \Phi_{k}\left(s_{1}\right)} \psi \circ \Phi_{k}\left(s_{2}\right)\right| \geq c>0
$$

for $k$ large and this shows that

$$
\left|\psi \circ \Phi_{k}\left(s_{1}\right)-\psi \circ \Phi_{k}\left(s_{2}\right)\right| \leq \frac{\exp \left(c\left|s_{1}-s_{2}\right|\right)-1}{\exp \left(c\left|s_{1}-s_{2}\right|\right)+1} \leq c\left|s_{1}-s_{2}\right|
$$

This shows that $\left\{\Phi_{k}\right\}$ is equicontinuous on $S_{1}$ and hence there is a subsequence of $\left\{\Phi_{k}\right\}$ that converges uniformly on compact subsets of $\Sigma_{z}$ to a continuous mapping $\Phi: \Sigma_{z} \rightarrow \mathbb{C}^{n}$. 
It may be observed that the same proof works when $f: \Omega_{1} \rightarrow \Omega_{2}$ is an isometry in the inner-Carathéodory distance on the domains. Indeed, the process of defining the scaling does not depend on the intrinsic metric that is used as a distance function. Moreover the Carathéodory distance enjoys the same functorial properties as the Kobayashi distance and even the quantitative bounds used in (3.8) and (3.10) remain the same. Hence the same proof works verbatim for the inner-Carathéodory distance.

Proposition 3.3. Let $f: \Omega_{1} \rightarrow \Omega_{2}$ be an isometry in the Kobayashi distance on smoothly bounded strongly pseudoconvex domains $\Omega_{1}, \Omega_{2}$ in $\mathbb{C}^{n}$. Then the limit map map $\Phi: \Sigma_{z} \rightarrow \mathbb{C}^{n}$ constructed above satisfies:

(i) $\Phi\left(\Sigma_{z}\right) \subset \Sigma_{w}$

(ii) $\Phi: \Sigma_{z} \rightarrow \Sigma_{w}$ is a $C^{0}$-isometry for the Kobayashi distance.

The same conclusions hold when $f: \Omega_{1} \rightarrow \Omega_{2}$ is an isometry in the innerCarathéodory distance on the domains, provided the boundaries are at least $C^{3}$ smooth. In particular $\Phi: \Sigma_{z} \rightarrow \Sigma_{w}$ is an isometry in the inner-Carathéodory distance (which equals the Kobayashi distance) on $\Sigma_{z}$ and $\Sigma_{w}$.

Proof. Let $\Phi_{k}: \tilde{\Omega}_{1}^{k} \rightarrow \tilde{\Omega}_{2}^{k}$ be the sequence of scaled mappings as before. Without loss of generality we assume that $\Phi_{k} \rightarrow \Phi$ uniformly on compact subsets of $\Sigma_{z}$. The defining equations for $\tilde{\Omega}_{2}^{k}$ and $\tilde{\Omega}_{1}^{k}$ are respectively given by

$$
\tilde{\rho}_{k}^{\prime}(w)=\frac{1}{\sqrt{\delta}_{k}} \rho_{k}\left(\sqrt{\delta}_{k} \tilde{w}, \delta_{k} w_{n}\right), \quad \tilde{\rho}_{k}(z)=\frac{1}{\sqrt{\delta}_{k}} \rho_{k}\left(\sqrt{\delta}_{k} \tilde{z}, \delta_{k} z_{n}\right) .
$$

It is shown in [23] that these equations simplify as

$$
\tilde{\rho}_{k}^{\prime}(w)=2 \operatorname{Re} w_{n}+|\tilde{w}|^{2}+\tilde{B}_{k}(w), \quad \tilde{\rho}_{k}(w)=2 \operatorname{Re} z_{n}+|\tilde{z}|^{2}+\tilde{A}_{k}(w)
$$

in neighborhoods of the origin where

$$
\left|\tilde{B}_{k}(w)\right| \leq|w|^{2}\left(c \sqrt{\delta}_{k}+\eta\left(\delta_{k}|w|^{2}\right)\right), \quad\left|\tilde{A}_{k}(z)\right| \leq|z|^{2}\left(c \sqrt{\delta}_{k}+\eta\left(\delta_{k}|z|^{2}\right)\right)
$$

with $\eta(t)=o(1)$ as $t \rightarrow 0$ and $c>0$ is uniform for all $k$ large.

Fix a compact subset of $\Sigma_{z}$, say $S$. Then for $k \gg 1$ and $z \in S$

$$
2 \operatorname{Re}\left(\Phi_{k}\right)_{n}(z)+\left|\tilde{\Phi}_{k}(z)\right|^{2}+\tilde{B}_{k}\left(\Phi_{k}(z)\right)<0
$$

where

$$
\left|\tilde{B}_{k}\left(\Phi_{k}(z)\right)\right| \leq\left|\Phi_{k}(z)\right|^{2}\left(c \sqrt{\delta_{k}}+\eta\left(\delta_{k}\left|\Phi_{k}(z)\right|^{2}\right)\right) .
$$

By the previous proposition $\left\{\Phi_{k}\right\}$ is uniformly bounded on $S$ and hence $\delta_{k}\left|\Phi_{k}(z)\right|^{2}$ converges to 0 , with the result that $\left.\eta\left(\delta_{k}\left|\Phi_{k}(z)\right|^{2}\right)\right) \rightarrow 0$ as $k \rightarrow \infty$. Passing to the limit as $k \rightarrow \infty$ in (3.13) shows that

$$
2 \operatorname{Re} \Phi_{n}(z)+|\tilde{\Phi}(z)|^{2} \leq 0
$$


which means exactly that $\Phi(C) \subset \bar{\Sigma}_{w}$. Since $S \subset \Sigma_{z}$ is arbitrary it follows that $\Phi: \Sigma_{z} \rightarrow \bar{\Sigma}_{w}$. If $\Phi$ were known to be holomorphic it would follow at once by the maximum principle that $\Phi: \Sigma_{z} \rightarrow \Sigma_{w}$. However $\Phi$ is known to be just continuous.

Let $D \subset \Sigma_{z}$ be the set of all points $z$ such that $\Phi(z) \in \Sigma_{w}$. D is nonempty since $(0,-1) \in D$ as can be seen from (3.6) and the fact that $\Phi_{k}(0,-1)=$ $\left(0,-\gamma_{k} / \delta_{k}\right)$. Since $\Phi$ is continuous, $D$ is open in $\Sigma_{z}$.

Claim. It suffices to show that

$$
d_{\Sigma_{z}}^{K}\left(z_{1}, z_{2}\right)=d_{\Sigma_{w}}^{K}\left(\Phi\left(z_{1}\right), \Phi\left(z_{2}\right)\right)
$$

for $z_{1}, z_{2} \in D$. Indeed, if $z_{0} \in \partial D \cap \Sigma_{z}$, choose a sequence $z_{j} \in D$ that converges to $z_{0}$. If the claim were true, then

$$
d_{\Sigma_{z}}^{K}\left(z_{j},(0,-1)\right)=d_{\Sigma_{w}}^{K}\left(\Phi\left(z_{j}\right), \Phi(0,-1)\right)
$$

for all $j$. Since $z_{0} \in \partial D, \Phi\left(z_{j}\right) \rightarrow \partial \Sigma_{w}$ and as $\Sigma_{w}$ is complete in the Kobayashi distance, the right side in (3.15) becomes unbounded. However the left side remains bounded, again because of the completeness of $\Sigma_{z}$. This contradiction would show that $D=\Sigma_{z}$, knowing which the claim would prove assertion ( $i i$ ) as well.

It is already known that

$$
d_{\tilde{\Omega}_{1}^{k}}^{K}\left(z_{1}, z_{2}\right)=d_{\tilde{\Omega}_{2}^{k}}^{K}\left(\Phi\left(z_{1}\right), \Phi\left(z_{2}\right)\right)
$$

for $k \gg 1$. To prove the claim it suffices to take limits on both sides in the equality above. This is an issue of the stability of the Kobayashi distance, to understand which we need to study the behaviour of the infinitesimal Kobayashi metric $\Phi_{\tilde{\Omega}_{1}^{k}}(z, v)$ as $k \rightarrow \infty$. To do this, we will use ideas from [13]. Once this is done, an integration argument will yield information about the global metric $K_{\tilde{\Omega}_{1}^{k}}$.

Step 1. It will be shown that

$$
\lim _{k \rightarrow \infty} F_{\tilde{\Omega}_{1}^{k}}^{K}(a, v)=F_{\Sigma_{z}}^{K}(a, v)
$$

for $(a, v) \in \Sigma_{z} \times \mathbb{C}^{n}$. Moreover, the convergence is uniform on compact subsets of $\Sigma_{z} \times \mathbb{C}^{n}$.

Let $S \subset \Sigma_{z}$ and $G \subset \mathbb{C}^{n}$ be compact and suppose that the desired convergence does not occur. Then there are points $a_{k} \in S$ converging to $a \in S$ and vectors $v_{k} \in G$ converging to $v \in G$ such that

$$
0<\varepsilon_{0}<\left|F_{\tilde{\Omega}_{1}^{k}}^{K}\left(a_{j}, v_{j}\right)-F_{\Sigma_{z}}^{K}\left(a_{j}, v_{j}\right)\right|
$$

for $j$ large. This inequality holds for a subsequence only, which will again be denoted by the same symbols. Further, since the infinitesimal metric is homogeneous of degree one in the vector variable, we can assume that $\left|v_{j}\right|=1$ for all $j$. It was 
proved in [13] that $F_{\Sigma_{z}}^{K}$ is jointly continuous in $(z, v)$. This was a consequence of the fact that $\Sigma_{z}$ is taut. Thus

$$
0<\varepsilon_{0} / 2<\left|F_{\tilde{\Omega}_{1}^{k}}^{K}\left(a_{j}, v_{j}\right)-F_{\Sigma_{z}}^{K}(a, v)\right| .
$$

The tautness of $\Sigma_{z}$ implies, via a normal families argument, that $0<F_{\Sigma_{z}}^{K}(a, v)<$ $\infty$ and there exists a holomorphic extremal disc $\phi: \Delta \rightarrow \Sigma_{z}$ that by definition satisfies $\phi(0)=a, \phi^{\prime}(0)=\mu v$ where $\mu>0$ and $F_{\Sigma}^{K}(a, v)=1 / \mu$.

Fix $\delta \in(0,1)$ and define the holomorphic maps $\phi_{k}: \Delta \rightarrow \mathbb{C}^{n}$ by

$$
\phi_{k}(\xi)=\phi((1-\delta) \xi)+\left(a_{k}-a\right)+\mu(1-\delta) \xi\left(v_{k}-v\right) .
$$

Observe that the image $\phi((1-\delta) \xi)$ is compact in $\Sigma_{z}$ and since $a_{k} \rightarrow a, v_{k} \rightarrow v$ it follows that $\phi_{k}: \Delta \rightarrow \tilde{\Omega}_{1}^{k}$ for $k$ large. Also, $\phi_{k}(0)=\phi(0)+a_{k}-a=a_{k}$ and that $\phi_{k}^{\prime}(0)=(1-\delta) \phi^{\prime}(0)+\mu(1-\delta)\left(v_{k}-v\right)=\mu(1-\delta)\left(v+v_{k}-v\right)=\mu(1-\delta) v_{k}$.

By the definition of the infinitesimal metric it follows that

$$
F_{\tilde{\Omega}_{1}^{k}}\left(a_{k}, v_{k}\right) \leq \frac{1}{\mu(1-\delta)}=\frac{F_{\Sigma_{z}}^{K}(a, v)}{1-\delta}
$$

for $j \gg 1$. Letting $\delta \rightarrow 0^{+}$it follows that

$$
\limsup _{k \rightarrow \infty} F_{\tilde{\Omega}_{1}^{k}}^{K}\left(a_{k}, v_{k}\right) \geq F_{\Sigma_{z}}^{K}(a, v) .
$$

Conversely, fix $\varepsilon>0$ arbitrarily small. By definition, there are holomorphic mappings $\phi_{k}: \Delta \rightarrow \tilde{\Omega}_{1}^{k}$ satisfying $\phi_{k}(0)=a_{k}, \phi_{k}^{\prime}(0)=\mu_{k} v_{k}$ where $\mu_{k}>0$ and

$$
F_{\tilde{\Omega}_{1}^{k}}^{K}\left(a_{k}, v_{k}\right) \geq \frac{1}{\mu_{k}}-\varepsilon .
$$

The sequence $\left\{\phi_{k}\right\}$ has a subsequence that converges to a holomorphic mapping $\phi: \Delta \rightarrow \Sigma_{z}$ uniformly on compact subsets of $\Delta$. Indeed consider the disc $\Delta_{r}$ of radius $r \in(0,1)$. The mappings $H_{k}^{-1} \circ \phi_{k}: \Delta \rightarrow \Omega_{1}$ and $H_{k}^{-1} \circ \phi_{k}(0) \rightarrow p \in \partial \Omega_{1}$. Fix a ball $B_{p}(\delta)$ of radius $\delta$ around $p$, with $\delta$ small enough. Since $p \in \partial \Omega_{1}$ is a plurisubharmonic peak point, Proposition 5.1 in [27] (see [3] also, where this phenomenon was aptly termed the attraction property of analytic discs) shows that for the value of $r \in(0,1)$ fixed earlier, there exists $\eta>0$, independent of $\phi_{k}$ such that $H_{k}^{-1} \circ \phi_{k}\left(\Delta_{r}\right) \subset B_{p}(\delta)$. If $\delta$ is small enough, then there exists $R>1$ large enough so that

$$
h_{k}\left(B_{p}(\delta) \cap \Omega_{1}\right) \subset\left\{z \in \mathbb{C}^{n}:\left|z_{n}+R\right|^{2}+|\tilde{z}|^{2}<R^{2}\right\} \subset \Omega .
$$

where (as in Proposition 3.2)

$$
\Omega=\left\{z \in \mathbb{C}^{n}: 2 R\left(\operatorname{Re} z_{n}\right)<-|\tilde{z}|^{2}\right\} .
$$


Again, we note that $\Omega$ is invariant under $T_{k}$ and that $\Omega \cong \mathbb{B}^{n}$. Hence $\phi_{k}\left(\Delta_{r}\right) \subset \Omega$ for $k$ large and this exactly means that

$$
2 R\left(\operatorname{Re}\left(\phi_{k}\right)_{n}(z)\right)+\left|\tilde{\phi}_{k}(z)\right|^{2}<0
$$

for $z \in \Delta_{r}$.

It follows that $\left\{\left(\phi_{k}\right)_{n}(z)\right\}$ and hence each component of $\tilde{\phi}_{k}(z)$, forms a normal family on $\Delta_{r}$. Since $r \in(0,1)$ was arbitrary, the usual diagonal subsequence yields a holomorphic mapping $\phi: \Delta \rightarrow \mathbb{C}^{n}$ or $\phi \equiv \infty$ on $\Delta$. But it is not possible that $\phi \equiv \infty$ on $\Delta$ since $\phi(0) \rightarrow a$.

It remains to show that $\phi: \Delta \rightarrow \Sigma_{z}$. For this note that $\tilde{\Omega}_{1}^{k}$ are defined by

$$
\tilde{\rho}_{k}(w)=2 \operatorname{Re} z_{n}+|\tilde{z}|^{2}+\tilde{A}_{k}(w),
$$

where

$$
\left|\tilde{A}_{k}(z)\right| \leq|z|^{2}\left(c \sqrt{\delta}_{k}+\eta\left(\delta_{k}|z|^{2}\right)\right)
$$

Thus for $\zeta \in \Delta_{r}, r \in(0,1)$,

$$
2 R\left(\operatorname{Re}\left(\phi_{k}\right)_{n}(z)+\left|\tilde{\phi}_{k}(z)\right|^{2}+\tilde{A}_{k}\left(\phi_{k}(z)\right)<0\right.
$$

where

$$
\tilde{A}_{k}\left(\phi_{k}(z)\right) \leq\left|\phi_{k}(z)\right|^{2}\left(c \sqrt{\delta}_{k}+\eta\left(\delta_{k}\left|\phi_{k}(z)\right|^{2}\right)\right)
$$

as $k \rightarrow \infty$. Passing to the limit in (3.19) shows that

$$
2 R\left(\operatorname{Re}\left(\phi_{k}\right)_{n}(z)\right)+\left|\tilde{\phi}_{k}(z)\right|^{2} \leq 0
$$

for $z \in \Delta_{r}$, which exactly means that $\phi\left(\Delta_{r}\right) \subset \bar{\Sigma}_{z}$. Since $r \in(0,1)$ was arbitrary, it follows that $\phi(\Delta) \subset \bar{\Sigma}_{z}$ and the maximum principle shows that $\phi(\Delta) \subset \Sigma_{z}$.

Note that $\phi(0)=a$ and $\phi^{\prime}(0)=\lim _{k \rightarrow \infty} \phi_{k}^{\prime}(0)=\lim _{k \rightarrow \infty} \mu_{k} v_{k}=\mu v$ for some $\mu>0$. It follows from (3.18) that

$$
\liminf _{k \rightarrow \infty} F_{\tilde{\Omega}_{1}^{k}}^{K}\left(a_{k}, v_{k}\right) \geq F_{\Sigma_{z}}^{K}(a, v)-\varepsilon .
$$

Combining (3.18) and (3.20) shows that

$$
\lim _{k \rightarrow \infty} F_{\tilde{\Omega}_{1}^{k}}^{K}\left(a_{k}, v_{k}\right)=F_{\Sigma_{z}}^{K}(a, v)
$$

which contradicts the assumption made and proves (3.16).

Step 2. The goal will now be to integrate (3.16) to recover the behaviour of the global metric, i.e., the distance function.

Let $\gamma:[0,1] \rightarrow \Sigma_{z}$ be a $C^{1}$ curve such that $\gamma(0)=z_{1}$ and $\gamma(0)=z_{2}$ and

$$
d_{\Sigma_{k}}^{K}\left(z_{1}, z_{2}\right)=\int_{0}^{1} F_{\Sigma_{z}}^{K}\left(\gamma, \gamma^{\prime}\right) \mathrm{d} t
$$


Since $\gamma \Subset \Sigma_{z}, \gamma \Subset \tilde{\Omega}_{1}^{k}$ for $k$ large. By Step 1, it follows that

$$
\int_{0}^{1} F_{\tilde{\Omega}_{1}^{k}}^{K}\left(\gamma, \gamma^{\prime}\right) \mathrm{d} t \leq \int_{0}^{1} F_{\Sigma_{z}}^{K}\left(\gamma, \gamma^{\prime}\right) \mathrm{d} t+\varepsilon=d_{\Sigma_{z}}^{K}\left(z_{1}, z_{2}\right)+\varepsilon
$$

By definition of $K_{\tilde{\Omega}_{1}^{k}}\left(z_{1}, z_{2}\right)$ it follows that

$$
d_{\tilde{\Omega}_{1}^{k}}^{K}\left(z_{1}, z_{2}\right) \leq \int_{0}^{1} F_{\tilde{\Omega}_{1}^{k}}^{K}\left(\gamma, \gamma^{\prime}\right) \mathrm{d} t \leq d_{\Sigma_{z}}^{K}\left(z_{1}, z_{2}\right)+\varepsilon .
$$

Thus

$$
\limsup _{k \rightarrow \infty} d_{\tilde{\Omega}_{1}^{k}}^{K}\left(z_{1}, z_{2}\right) \leq d_{\Sigma_{z}}^{K}\left(z_{1}, z_{2}\right)
$$

Conversely since $z_{1}, z_{2} \in D \subset \Sigma_{z}$, it follows that $z_{1}, z_{2} \in \tilde{\Omega}_{1}^{k}$ for $k$ large. Fix $\varepsilon>0$ and let $B_{p}\left(\eta_{1}\right)$ be a small enough neighbourhood of $p \in \partial \Omega$. Choose $\eta_{2}<\eta_{1}$ so that

$$
F_{\Omega_{1}}^{K}(z, v) \leq F_{B_{p}\left(\eta_{1}\right) \cap \Omega_{1}}^{K}(z, v) \leq(1+\varepsilon) F_{\Omega_{1}}^{K}(z, v)
$$

for $z \in B_{p}\left(\eta_{2}\right) \cap \Omega_{1}$ and $v$ a tangent vector at $z$. This is possible by the localization property of the Kobayashi metric near strongly pseudoconvex points.

If $k$ is sufficiently large, $H_{k}^{-1}\left(z_{1}\right)$ and $H_{k}^{-1}\left(z_{1}\right)$ both belong to $B_{p}\left(\eta_{2}\right) \cap \Omega_{1}$. If $\eta_{1}$ is small enough, $B_{p}\left(\eta_{1}\right) \cap \Omega_{1}$ is strictly convex and it follows from Lempert's work that there exist $m_{k}>1$ and holomorphic mappings

$$
\phi_{k}: \Delta_{m_{k}} \rightarrow B_{p}\left(\eta_{1}\right) \cap \Omega_{1}
$$

such that $\phi_{k}(0)=H_{k}^{-1}\left(z_{1}\right), \phi_{k}(1)=H_{k}^{-1}\left(z_{2}\right)$ and

$$
\begin{aligned}
d_{B_{p}\left(\eta_{1}\right) \cap \Omega_{1}}^{K}\left(H_{k}^{-1}\left(z_{1}\right), H_{k}^{-1}\left(z_{2}\right)\right) & =\rho_{\Delta_{m_{k}}}(0,1) \\
& =\int_{0}^{1} F_{B_{p}\left(\eta_{1}\right) \cap \Omega_{1}}^{K}\left(\phi_{k}(t), \phi_{k}^{\prime}(t)\right) \mathrm{d} t .
\end{aligned}
$$

Integrating (3.22) and using the fact that $H_{k}$ are biholomorphisms and hence Kobayashi isometries, it follows that

$$
d_{H_{k}\left(B_{p}\left(\eta_{1}\right) \cap \Omega_{1}\right)}^{K}\left(z_{1}, z_{2}\right) \leq(1+\varepsilon) d_{\tilde{\Omega}_{1}^{k}}^{K}\left(z_{1}, z_{2}\right) .
$$

Hence (3.23) shows that

$$
\begin{aligned}
\frac{1}{2} \log \left(\frac{m_{k}+1}{m_{k}-1}\right)=\rho_{\Delta_{m_{k}}}(0,1) & =d_{B_{p}\left(\eta_{1}\right) \cap \Omega_{1}}^{K}\left(H_{k}^{-1}\left(z_{1}\right), H_{k}^{-1}\left(z_{2}\right)\right) \\
& =d_{H_{k}\left(B_{p}\left(\eta_{1}\right) \cap \Omega_{1}\right)}^{K}\left(z_{1}, z_{2}\right) \leq(1+\varepsilon) d_{\tilde{\Omega}_{1}^{k}}^{K}\left(z_{1}, z_{2}\right) .
\end{aligned}
$$


But

$$
d_{\tilde{\Omega}_{1}^{k}}^{K}\left(z_{1}, z_{2}\right) \leq d_{\Sigma_{z}}^{K}\left(z_{1}, z_{2}\right)+\varepsilon<\infty
$$

and hence $m_{k}>1+\delta$ for some uniform $\delta>0$ for all $k \gg 1$. Thus the holomorphic mappings $\sigma_{k}:=T_{k} \circ h_{k} \circ \phi_{k}: \Delta_{1+\delta} \rightarrow T_{k} \circ h_{k}\left(U \cap \Omega_{1}\right) \subset \tilde{\Omega}_{1}^{k}$ are well-defined and satisfy $\sigma_{k}(0)=z_{1}$ and $\sigma_{k}(1)=z_{2}$. Now exactly the same arguments that were used to establish the lower semi-continuity of the infinitesimal metric in Step 1 show that $\left\{\sigma_{k}\right\}$ is a normal family and $\sigma_{k} \rightarrow \sigma: \Delta_{1+\delta} \rightarrow \Sigma_{z}$ uniformly on compact subsets of $\Delta_{1+\delta}$. Again using (3.22) and (3.23) we get

$$
\begin{aligned}
\int_{0}^{1} F_{\tilde{\Omega}_{1}^{k}}^{K}\left(\sigma_{k}, \sigma_{k}^{\prime}\right) \mathrm{d} t \leq \int_{0}^{1} F_{H_{k}\left(B_{p}\left(\eta_{1}\right) \cap \Omega_{1}\right)}^{K}\left(\sigma_{k}, \sigma_{k}^{\prime}\right) \mathrm{d} t & =d_{H_{k}\left(B_{p}\left(\eta_{1}\right) \cap \Omega_{1}\right)}^{K}\left(z_{1}, z_{2}\right) \\
& \leq(1+\varepsilon) d_{\tilde{\Omega}_{1}^{k}}^{K}\left(z_{1}, z_{2}\right) .
\end{aligned}
$$

Since $\sigma_{k} \rightarrow \sigma$ and $\sigma_{k}^{\prime} \rightarrow \sigma^{\prime}$ uniformly on $[0,1]$, Step 1 shows that

$$
\int_{0}^{1} F_{\Sigma_{z}}^{K}\left(\sigma, \sigma^{\prime}\right) \mathrm{d} t \leq \int_{0}^{1} F_{\tilde{\Omega}_{1}^{k}}^{K}\left(\sigma_{k}, \sigma_{k}^{\prime}\right) \mathrm{d} t+\varepsilon \leq d_{\Sigma_{z}}^{K}\left(z_{1}, z_{2}\right)+C \varepsilon
$$

for all large $k$.

It remains to note that since $\sigma(t), 0 \leq t \leq 1$ joins $z_{1}, z_{2}$ it follows that

$$
d_{\Sigma_{z}}^{K}\left(z_{1}, z_{2}\right) \leq \int_{0}^{1} F_{\tilde{\Omega}_{1}^{k}}^{K}\left(\sigma_{k}, \sigma_{k}^{\prime}\right) \mathrm{d} t \leq d_{\tilde{\Omega}_{1}^{k}}^{K}\left(z_{1}, z_{2}\right)+C \varepsilon .
$$

Combining (3.21) and (3.24), we see that

$$
d_{\tilde{\Omega}_{1}^{k}}^{K}\left(z_{1}, z_{2}\right) \rightarrow d_{\Sigma_{z}}^{K}\left(z_{1}, z_{2}\right)
$$

for all $z_{1}, z_{2} \in D$. Exactly the same arguments show that it is possible to pass to the limit on the right side of (3.14). The claim made in (3.14) follows.

To complete the proof of the proposition for the Kobayashi distance, it remains to show that $\Phi: \Sigma_{z} \rightarrow \Sigma_{w}$ is surjective. This follows by repeating the argument of the previous proposition for $f^{-1}: \Omega_{2} \rightarrow \Omega_{1}$ and considering the scaled inverses, i.e. $\Psi_{k}=\Phi_{k}^{-1}: \tilde{\Omega}_{2}^{k} \rightarrow \tilde{\Omega}_{1}^{k}$. This family will converge to a continuous map $\Psi: \Sigma_{w} \rightarrow \mathbb{C}^{n}$ uniformly on compact subsets of $\Sigma_{w}$. The arguments of this proposition will then show that $\Psi$ maps $\Sigma_{w}$ to $\Sigma_{z}$. Finally observe that for $w$ in a fixed compact subset of $\Sigma_{w}$,

$$
\begin{aligned}
|w-\Phi \circ \Psi(w)| & =\left|\Phi_{k} \circ \Psi_{k}(w)-\Phi \circ \Psi(w)\right| \\
& \leq\left|\Phi_{k} \circ \Psi_{k}(w)-\Phi \circ \Psi_{k}(w)\right|+\left|\Phi \circ \Psi_{k}(w)-\Phi \circ \Psi(w)\right| \rightarrow 0
\end{aligned}
$$

as $k \rightarrow \infty$. Thus $\Phi \circ \Psi=i d=\Psi \circ \Phi$. 
We now deal with the case when $f: \Omega_{1} \rightarrow \Omega_{2}$ is an isometry for the innerCarthéodory distance on $\Omega_{1}$ and $\Omega_{2}$.

One possibility is to first show that

$$
\lim _{k \rightarrow \infty} d_{\tilde{\Omega}_{1}^{k}}^{C}\left(z_{1}, z_{2}\right)=d_{\Sigma_{z}}^{C}\left(z_{1}, z_{2}\right)
$$

for $z_{1}, z_{2} \in \Sigma_{z}$. Knowing this, the following inequalities hold:

$$
\begin{aligned}
d_{\Sigma_{z}}^{C}\left(z_{1}, z_{2}\right)=\lim _{k \rightarrow \infty} d_{\tilde{\Omega}_{1}^{k}}^{C}\left(z_{1}, z_{2}\right) & \leq \lim _{k \rightarrow \infty} d_{\tilde{\Omega}_{1}^{k}}^{\tilde{C}}\left(z_{1}, z_{2}\right) \\
& \leq \lim _{k \rightarrow \infty} d_{\tilde{\Omega}_{1}^{k}}^{K}\left(z_{1}, z_{2}\right)=d_{\Sigma_{z}}^{K}\left(z_{1}, z_{2}\right) .
\end{aligned}
$$

Since $d_{\Sigma_{z}}^{C}=d_{\Sigma_{z}}^{\tilde{C}}=d_{\Sigma_{z}}^{K}$, it would follow that

$$
d_{\Sigma_{z}}^{\tilde{C}}\left(z_{1}, z_{2}\right)=\lim _{k \rightarrow \infty} d_{\tilde{\Omega}_{1}^{k}}^{\tilde{C}}\left(z_{1}, z_{2}\right)
$$

Hence it suffices to show the stability of the Carathéodory distance.

As before let $z_{1}, z_{2} \in S \Subset \Sigma_{z}$. For large $k, z_{1}, z_{2} \in \tilde{\Omega}_{1}^{k}$. Let $\phi_{k}: \tilde{\Omega}_{1}^{k} \rightarrow \Delta$ be holomorphic maps such that $\phi_{k}\left(z_{1}\right)=0$ and

$$
d_{\tilde{\Omega}_{1}^{k}}^{C}\left(z_{1}, z_{2}\right)=\rho\left(0, \phi_{k}\left(z_{2}\right)\right) .
$$

The family $\left\{\phi_{k}\right\}$ is uniformly bounded above and since $\tilde{\Omega}_{1}^{k} \rightarrow \Sigma_{z}$, all mappings $\phi_{k}$, $k \geq k_{0}$, are defined on the compact set $S$. Thus there is a subsequence which will still be denoted by $\phi_{k}$ so that $\phi_{k} \rightarrow \phi: \Sigma_{z} \rightarrow \bar{\Delta}$ and $\phi\left(z_{1}\right)=0$. If $\left|\phi\left(z_{0}\right)\right|=1$ for some $z_{0} \in \Sigma_{z}$, then $|\phi(z)| \equiv 1$ by the maximum principle. Thus $\phi: \Sigma_{z} \rightarrow \Delta$ and in particular $\rho\left(0, \phi_{k}\left(z_{2}\right)\right) \rightarrow \rho\left(0, \phi\left(z_{2}\right)\right)$. Therefore $d_{\tilde{\Omega}_{1}^{k}}^{C}\left(z_{1}, z_{2}\right) \leq \rho\left(0, \phi\left(z_{2}\right)\right)+$ $\varepsilon \leq d_{\Sigma_{z}}^{C}\left(z_{1}, z_{2}\right)+\varepsilon$, which shows that

$$
\limsup _{k \rightarrow \infty} d_{\tilde{\Omega}_{1}^{k}}^{C}\left(z_{1}, z_{2}\right) \leq d_{\Sigma_{z}}^{C}\left(z_{1}, z_{2}\right)
$$

Conversely, working with the same subsequence that was extracted above, we have:

$$
d_{\Sigma_{z}}^{C}\left(z_{1}, z_{2}\right) \leq d_{\Sigma_{z}}^{K}\left(z_{1}, z_{2}\right) \leq d_{\tilde{\Omega}_{1}^{k}}^{C}\left(z_{1}, z_{2}\right)+\varepsilon
$$

for $k$ large. But

$$
d_{\tilde{\Omega}_{1}^{k}}^{K}\left(z_{1}, z_{2}\right)=d_{\Omega_{1}}^{K}\left(H_{k}^{-1}\left(z_{1}\right), H_{k}^{-1}\left(z_{2}\right)\right)
$$

and since $H_{k}^{-1}\left(z_{1}\right), H_{k}^{-1}\left(z_{2}\right)$ are both close to $p$, for $k$ large, it follows that

$$
d_{\Omega_{1}}^{K}\left(H_{k}^{-1}\left(z_{1}\right), H_{k}^{-1}\left(z_{2}\right)\right) \leq d_{B_{p}\left(\eta_{1}\right) \cap \Omega_{1}}^{K}\left(H_{k}^{-1}\left(z_{1}\right), H_{k}^{-1}\left(z_{2}\right)\right)+\varepsilon
$$


where $B_{p}\left(\eta_{1}\right)$ is a small enough neighborhood of $p$. Since $B_{p}\left(\eta_{1}\right) \cap \Omega_{1}$ is convex Lempert's work shows that the Kobayashi and Carthéodory distances coincide. Combining the aforementioned observation, we get

$$
d_{\Sigma_{z}}^{C}\left(z_{1}, z_{2}\right) \leq d_{B_{p}\left(\eta_{1}\right) \cap \Omega_{1}}^{C}\left(H_{k}^{-1}\left(z_{1}\right), H_{k}^{-1}\left(z_{2}\right)\right)+2 \varepsilon
$$

To conclude, it is known (see [23]) that the Carathéodory distance can be localised near strongly pseudoconvex points, exactly like the Kobayashi distance. hence

$$
\begin{aligned}
d_{B_{p}\left(\eta_{1}\right) \cap \Omega_{1}}^{C}\left(H_{k}^{-1}\left(z_{1}\right), H_{k}^{-1}\left(z_{2}\right)\right) & \leq(1+\varepsilon) d_{\Omega_{1}}^{C}\left(H_{k}^{-1}\left(z_{1}\right), H_{k}^{-1}\left(z_{2}\right)\right) \\
& =(1+\varepsilon) d_{\tilde{\Omega}_{1}^{k}}^{C}\left(z_{1}, z_{2}\right)
\end{aligned}
$$

With this (3.26) becomes

$$
d_{\Sigma_{z}}^{C}\left(z_{1}, z_{2}\right) \leq(1+\varepsilon) d_{\tilde{\Omega}_{1}^{k}}^{C}\left(z_{1}, z_{2}\right)+2 \varepsilon
$$

Since $d_{\tilde{\Omega}_{1}^{k}}^{C}\left(z_{1}, z_{2}\right)$ are uniformly bounded by (3.25) it follows that

$$
d_{\Sigma_{z}}^{C}\left(z_{1}, z_{2}\right) \leq d_{\tilde{\Omega}_{1}^{k}}^{C}\left(z_{1}, z_{2}\right)+C \varepsilon
$$

combining (3.25) and (3.28), we see that

$$
\lim _{k \rightarrow \infty} d_{\tilde{\Omega}_{1}^{k}}^{C}\left(z_{1}, z_{2}\right)=d_{\Sigma_{z}}^{C}\left(z_{1}, z_{2}\right) .
$$

Hence the claim made in (3.14) also holds for the inner-Carathéodory metric. The concluding arguments remain the same in this case as well. This completes the proof of the proposition.

Since the Kobayashi and Caratheodory distances coincide with a constant multiple of the Bergman metric on $\Sigma_{z}$ and $\Sigma_{w}$, it follows from [12] that the limit map $\Phi: \Sigma_{z} \rightarrow \Sigma_{w}$ is (anti)-biholomorphic.

\section{The boundary map is CR/anti-CR}

We prove Theorem 1.1 in this section. Throughout, $f: \Omega_{1} \rightarrow \Omega_{2}$ will denote a $C^{1}$ isometry of the Kobayashi or inner-Carathéodory metrics which has a $C^{1}$-extension to $\bar{\Omega}_{1}$.

Fix $p \in \partial \Omega_{1}$. For the rest of this section we assume that $p=f(p)=0$ and that the real normals to $\Omega_{1}$ and $\Omega_{2}$ at $p$ and $f(p)$ are given by $\left\{\tilde{z}=\operatorname{Im} z_{n}=0\right\}$ and $\left\{\tilde{w}=\operatorname{Im} w_{n}=0\right\}$. This can be achieved by composing $f$ with transformations of the type in Lemma 3.1. 
Fix a sequence $\delta_{k} \rightarrow 0$ and define $x_{k} \in \Omega_{1}$ by $x_{k}=\left(\tilde{0},-\delta_{k}\right)$. Then $x_{k} \rightarrow p$ as $k \rightarrow \infty$. Because of our choice of $x_{k}$, in the notation of Section 3, the map $h_{k}=i d$. Recall that $f_{k}=g_{k} \circ f$ and $\Phi_{k}=T_{k} \circ f_{k} \circ T_{k}^{-1}$. More explicitly

$$
\Phi_{k}(z)=\left(\frac{1}{\sqrt{\delta}_{k}} \tilde{f}_{k}\left(\sqrt{\delta}_{k} \tilde{z}, \delta_{k} z_{n}\right), \frac{1}{\delta_{k}}\left(f_{k}\right)_{n}\left(\sqrt{\delta}_{k} \tilde{z}, \delta_{k} z_{n}\right)\right)
$$

Lemma 4.1. For any $\alpha \in \mathbb{C}$ such that $\operatorname{Re} \alpha<0,\left|\tilde{f}_{k}\left(0, \delta_{k} \alpha\right)\right|=O\left(\delta_{k}\right)$ as $k \rightarrow \infty$.

Proof. Let $m$ be an upper bound for $|d f|$ on $\bar{\Omega}_{1}$. The condition on $\alpha$ just ensures that $\left(0, \delta_{k} \alpha\right)$ lies in $\Omega_{1}^{k}$, which is the domain of $f_{k}$.

Now, noting that $\tilde{f}_{k}\left(x_{k}\right)=0$ and $|\tilde{v}| \leq|v|$ for any $v \in \mathbb{C}^{n}$, we have

$$
\begin{aligned}
\left|\tilde{f}_{k}\left(0, \delta_{k} \alpha\right)\right| & =\left|\tilde{f}_{k}\left(x_{k}\right)-\tilde{f}_{k}\left(0, \delta_{k} \alpha\right)\right| \leq\left|f_{k}\left(x_{k}\right)-f_{k}\left(0, \delta_{k} \alpha\right)\right| \\
& \leq C\left|f\left(x_{k}\right)-f\left(0, \delta_{k} \alpha\right)\right| \leq C m\left|x_{k}-\left(0, \delta_{k} \alpha\right)\right| \\
& =C m|\alpha+1| \delta_{k}
\end{aligned}
$$

since $x_{k}=\left(0,-\delta_{k}\right)$. The second inequality above can be explained as follows: First, $g_{k} \rightarrow i d$ in $C^{\infty}$ on compact subsets of $\mathbb{C}^{n}$. Hence on any compact set which contains 0 , there is a constant $C$ such that $\left|d g_{k}\right| \leq C$ for large $k$. Then an application of the mean-value inequality completes the argument.

The next lemma provides the crucial link between the limit of the re-scaled isometries and the derivative of the boundary map. We clarify the notation used in the statement and proof: First, even when we use complex notation, all quantities will be regarded as entities on real Euclidean space. In particular $\mathbb{C}^{n}$ is identified with $\mathbb{R}^{2 n}$ by $z=\left(z_{1}, \ldots, z_{n}\right)=\left(x_{1}+\sqrt{-1} x_{2}, \ldots, x_{2 n-1}+\sqrt{-1} x_{2 n}\right) \equiv\left(x_{1}, \ldots, x_{2 n}\right)$. Second, by the normalizations made at the beginning of this section we note that, at $p$, the decomposition $T_{p} \Omega_{1}=\mathbb{C}^{n}=H_{p}\left(\partial \Omega_{1}\right) \oplus H_{p}\left(\partial \Omega_{1}\right)^{\perp}$ coincides with $\mathbb{C}^{n}=\mathbb{C}^{n-1} \oplus \mathbb{C}$. Hence, by an abuse of notation, for $v \in T_{p} \Omega_{1}$, if $v=v_{H}+v_{N}$ then $v=\left(v_{H}, v_{N}\right)$. Similar remarks hold for $f(p)$ and $\Omega_{2}$.

Next, by Proposition 3.3, a subsequence of $\left\{\Phi_{k}\right\}$ converges to a (anti)-holomorphic automorphism $\Phi: \Sigma_{z} \rightarrow \Sigma_{w}$. For the statement of the lemma it helps to regard $\Sigma_{z}$ and $\Sigma_{w}$ as subsets of $T_{p} \Omega_{1}$ and $T_{f(p)} \Omega_{2}$ respectively.

Lemma 4.2. With notation as above, for any $z=\left(\tilde{z}, z_{n}\right) \in \Sigma_{z}$, we have $\widetilde{d f}(\tilde{z}, 0)=$ $d \tilde{f}_{p}(\tilde{z}, 0)=\tilde{\Phi}(z)$.

Proof. The first equality is clear from the definitions. As for the second, consider any map $r: \mathbb{C}^{n} \rightarrow \mathbb{C}^{n-1}$ with $r=\left(r_{1}, \ldots, r_{n-1}\right)$. Given $\delta$ and $a \in \mathbb{C}$, we write

$$
\frac{1}{\sqrt{\delta}} r\left(\sqrt{\delta} \tilde{z}, \delta z_{n}\right)=\frac{r\left(\sqrt{\delta} \tilde{z}, \delta z_{n}\right)-r\left(0, \delta z_{n}\right)}{\sqrt{\delta}}+\frac{r\left(0, \delta z_{n}\right)}{\sqrt{\delta}} .
$$


By using the mean value theorem for one-variable functions repeatedly, we can rewrite the above equation as

$$
\frac{1}{\sqrt{\delta}} r\left(\sqrt{\delta} \tilde{z}, \delta z_{n}\right)=N \tilde{z}+\frac{r\left(0, \delta z_{n}\right)}{\sqrt{\delta}} .
$$

Here $N$ is a real matrices of size $(2 n-2) \times(2 n-2)$ with entries $N_{i j}=\frac{\partial r_{i}}{\partial x_{j}}\left(\xi_{i j}(\delta)\right)$. Also, $\tilde{z}$ is regarded as a column vector of size $(2 n-2) \times 1$. The entries of $\xi_{i j}(\delta)$ lie between the entries of $\left(0, \delta z_{n}\right)$ and $\left(\sqrt{\delta} \tilde{z}, \delta z_{n}\right)$.

Now apply this to $r=\tilde{f}_{k}$ and $\delta=\delta_{k}$ and let $k \rightarrow \infty$. The only point to be noted is that since $\left(\tilde{z}, z_{n}\right) \in \Sigma_{z}$ by hypothesis, we have that $\left(\sqrt{\delta}_{k} \tilde{z}, \delta_{k} z_{n}\right)$ and $\left(0, \delta_{k} z_{n}\right)$ lie in $\Omega_{1}^{k}$ for large $k$. Since $\Omega_{1}^{k}$ is the domain of definition of $f_{k}$, the above equations make sense.

As $k \rightarrow \infty$ in (4.1), the second term on the right-hand side goes to zero by Lemma 4.1. Since $\left\{g_{k}\right\}$ converges to the identity map as $k \rightarrow \infty$, we have $\frac{\partial\left(\tilde{f}_{k}\right)_{i}}{\partial x_{j}}\left(\xi_{i j}^{k}\left(\delta_{k}\right)\right) \rightarrow \frac{\partial \tilde{f}_{i}}{\partial x_{j}}(0)$ by the continuity assumption of $d f$ on $\bar{\Omega}_{1}$. Hence the first term converges to $d \tilde{f}_{p}(\tilde{z}, 0)$.

To complete the proof, we observe that since $\Phi_{k} \rightarrow \Phi, \frac{1}{\sqrt{\delta}_{k}} \tilde{f}_{k}\left(\sqrt{\delta}_{k} \tilde{z}, \delta_{k} z_{n}\right) \rightarrow$ $\tilde{\Phi}(z)$.

We proceed with the proof of Theorem 1.1. Recall that if $\Omega_{1}$ and $\Omega_{2}$ are domains with smooth boundaries in $\mathbb{C}^{n}$, a $C^{1}$ map $\phi: \partial \Omega_{1} \rightarrow \partial \Omega_{2}$ is said to be $C R$ if, for every $p \in \partial \Omega_{1}$, the following two conditions are satisfied

$$
d \phi_{p}\left(H_{p}\left(\partial \Omega_{1}\right)\right) \subset H_{\phi(p)}\left(\partial \Omega_{2}\right)
$$

and

$$
d \phi_{p} \circ J_{1}=J_{2} \circ d \phi_{p}
$$

where $J_{1}$ and $J_{2}$ are the almost complex structures on $H_{p}\left(\partial \Omega_{1}\right)$ and $H_{\phi(p)}\left(\partial \Omega_{2}\right)$. Similarly, an anti-CR map satisfies $d \phi_{p}\left(H_{p}\left(\partial \Omega_{1}\right)\right) \subset H_{\phi(p)}\left(\partial \Omega_{2}\right)$ and $d \phi_{p} \circ J_{1}=$ $-J_{2} \circ d \phi_{p}$ for every $p \in \partial \Omega_{1}$.

In our case, $d f$ satisfies the first condition by Lemma 2.3. We claim the second condition is satisfied due to Lemma 4.2. It follows from this lemma that the map $T: \mathbb{B}^{n-1} \rightarrow \mathbb{C}^{n-1}$ given by $T(\tilde{z})=\tilde{\Phi}(\tilde{z},-1)$ is the restriction of the $\mathbb{R}$-linear map $\widetilde{d f_{p}}: \mathbb{C}^{n-1} \rightarrow \mathbb{C}^{n-1}$. On the other hand, $\tilde{\Phi}$ is holomorphic or anti-holomorphic (since $\Phi$ is so). Combining these two observations, it follows that $T$ is actually the restriction of a $\mathbb{C}$-linear map. Hence,

$$
\tilde{\Phi}\left(J_{1}(v),-1\right)= \pm J_{2} \tilde{\Phi}(v,-1),
$$

for any $v \in \mathbb{B}^{n-1}$ and where $J_{1}, J_{2}$ denote the almost-complex structures on $H_{p}\left(\partial \Omega_{1}\right)$ and $H_{\Phi(p)}\left(\partial \Omega_{2}\right)$ respectively (note that we have used the identification of the horizontal subspaces with $\left.\mathbb{C}^{n-1}\right)$. 
This implies that $d f_{p}$ is actually a $\mathbb{C}$-linear or conjugate linear map on $\mathbb{C}^{n-1}=$ $H_{p}\left(\partial \Omega_{1}\right)$. More explicitly, let $v \in H_{p}\left(\partial \Omega_{1}\right)$. By scaling $v$ by some constant $\alpha>0$, we can assume that $(\alpha v,-1) \in \Sigma_{z}$. By Lemmas 4.2 and 2.3, we have

$$
d f_{p}(\alpha v, 0)=(\tilde{\Phi}(\alpha v,-1), 0)
$$

Using the $\mathbb{C}$-linearity/conjugate-linearity of $\Phi$ as in (4.2), we have

$$
d f_{p}\left(J_{1}(\alpha v), 0\right)=\left(\tilde{\Phi}\left(J_{1}(\alpha v),-1\right), 0\right)= \pm\left(J_{2} \tilde{\Phi}(\alpha v,-1), 0\right)= \pm J_{2} d f_{p}(\alpha v, 0)
$$

Hence we conclude that the boundary map is CR/anti-CR.

Now we prove that $d f_{p}: T_{p} \partial \Omega_{1} \rightarrow T_{f(P)} \partial \Omega_{2}$ is an isomorphism. First, note that $\left.d f_{p}\right|_{H_{p}\left(\partial \Omega_{1}\right)}: H_{p}\left(\partial \Omega_{1}\right) \rightarrow H_{f(p)}\left(\partial \Omega_{2}\right)$ is invertible. To see this, let $\mathbb{H}_{z}:=\Sigma_{z} \cap\left\{\left(0, z_{n}\right): z_{n} \in \mathbb{C}\right\}$. Then $\Phi\left(\mathbb{H}_{z}\right) \subset \mathbb{H}_{w}$ by Lemma 4.2. On the other hand, it can be checked that the induced Riemannian metrics on $\mathbb{H}_{z}$ and $\mathbb{H}_{w}$ are just the hyperbolic metrics. From the completeness of these metrics it follows that $\Phi\left(\mathbb{H}_{z}\right)=\mathbb{H}_{w}$. But if $d f_{p}$ is not injective on $H_{p}\left(\partial \Omega_{1}\right)$, then there would be a $(v, 0) \in H_{p}\left(\partial \Omega_{1}\right)$ such that $d f_{p}(v, 0)=0$. By scaling $v$ we can assume that $(v,-1) \in \Sigma_{z}$ and use Lemma 4.2 to conclude that $\Phi(v,-1) \in \mathbb{H}_{w}$. This contradicts $\Phi^{-1}\left(\mathbb{H}_{w}\right)=\mathbb{H}_{z}$.

Next, as in the proof of Lemma 2.3, Equation (2.3) shows that $d f_{p}(v)_{N} \neq 0$ for any $v \in H_{p}\left(\partial \Omega_{1}\right)^{\perp}$. Hence $d f_{p}: T_{p} \partial \Omega_{1} \rightarrow T_{f(p)} \partial \Omega_{2}$ is invertible and $f$ is a $\mathrm{CR} /$ anti-CR diffeomorphism.

To conclude that $\Omega_{1}$ and $\Omega_{2}$ are biholomorphic we proceed as follows: Note that the connectedness of $\partial \Omega_{1}$ implies that $f: \partial \Omega_{1} \rightarrow \partial \Omega_{2}$ is either CR or anti$\mathrm{CR}$ everywhere. Let us assume that $f$ is $\mathrm{CR}$ everywhere, the other case being exactly similar. It follows that there is a neighborhood $U_{1}$ of $\partial \Omega_{1}$ and a holomorphic mapping $F: U_{1} \cap \Omega_{1} \rightarrow \Omega_{2}$ such that $F$ is $C^{1}$-smooth upto $\partial \Omega_{1}$ and $F=f$ on $\partial \Omega_{1}$. By Hartogs' theorem, $F$ extends to a holomorphic mapping $F: \Omega_{1} \rightarrow \Omega_{2}$. Similarly $f^{-1}$ has a holomorphic extension, say $G: \Omega_{2} \rightarrow \Omega_{1}$, which agrees with $f^{-1}$ on $\partial \Omega_{2}$. Since $f \circ f^{-1}=F \circ G=i d$ on $\partial \Omega_{2}$, the uniqueness theorem of [21] forces $F \circ G=i d$ on $\Omega_{2}$ and likewise $G \circ F=i d$ on $\Omega_{1}$. Thus $\Omega_{1}$ and $\Omega_{2}$ are biholomorphic.

\section{References}

[1] Z. M. BALOGH and M. BONK, Gromov hyperbolicity and the Kobayashi metric on strictly pseudoconvex domains, Comment. Math. Helv. 75 (2000), 504-533.

[2] Z. M. BALOGH and M. Bonk, Pseudoconvexity and Gromov hyperbolicity, C. R. Acad. Sci. Paris Sèr. I Math. 328(1999), 597-602.

[3] F. BertelOOT, Attraction des disques analytiques et continuitè höldérienne d'applications holomorphes propres, In: "Topics in Complex Analysis"(Warsaw, 1992), Banach Center Publ., Vol. 31, Polish Acad. Sci., Warsaw, 1995.

[4] J. Bland, T. Duchamp and M. KalKa, On the automorphism group of strictly convex domains in $\mathbb{C}^{n}$, In: "Complex Differential Geometry and Nonlinear Differential Equations" (Brunswick, maine, 1984), Comtemp. Math. Vol. 49, 1986, 19-30. 
[5] S. Y. CHENG and S. T. YAU, On the existence of a complete Kähler metric on noncompact complex manifolds and the regularity of Fefferman's equation, Comm. Pure Appl. Math. 33 (1980), 507-544.

[6] K. Diederich and S. PINCHUK, Proper holomorphic maps in dimension 2 extend, Indiana Univ. Math. J. 44 (1995), 1089-1125.

[7] K. DIEDERICH and S. WEBSTER, A reflection principle for degenerate real hypersurfaces, Duke Math. J. 47 (1980), 835-843.

[8] F. Forstneric, An elementary proof of Fefferman's theorem, Expo. Math. 10 (1992), 135149.

[9] H. Gaussier, K-T. KIM and S. G. Krantz, A note on the Wong-Rosay theorem in complex manifolds, Complex Variables Theory Appl. 47 (2002), 761-768.

[10] I. GRAham, Boundary behavior of the Carathèodory and Kobayashi metrics on strongly pseudoconvex domains in $\mathbb{C}^{n}$ with smooth boundary, Trans. Amer. Math. Soc. 207 (1975), 219-240.

[11] I. GRAHAM, Holomorphic mappings into strictly convex domains which are Kobayashi isometries at one point, Proc. Amer. Math. Soc. 105 (1989), 917-921.

[12] R. E. GREENE and S. G. KRANTZ, Deformation of complex structures, estimates for the $\bar{\partial}$ equation, and stability of the Bergman kernel, Adv. Math. 43 (1982), 1-86.

[13] R. E. GREENE and S. G. KRANTZ, Stability of the Carathèodory and Kobayashi metrics and applications to biholomorphic mappings, In: "Complex Analysis of Several Variables (Madison, Wis., 1982), 77-93, Proc. Sympos. Pure Math. 41, Amer. Math. Soc., Providence, RI, 1984.

[14] S. KobAYASHI, Intrinsic metrics on complex manifolds, Bull. Amer. Math. Soc. 73 (1967), 347-349.

[15] L. D. KAY, On the Kobayashi-Royden metric for ellipsoids, Math. Ann. 289 (1991), 55-72.

[16] L. LEMPERT, La métrique de Kobayashi et la représentation des domaines sur la boule, Bull. Soc. Math. France 109 (1981), 427-474.

[17] D. MA, On iterates of holomorphic maps, Math. Z. 207 (1991), 417-428.

[18] S. B. MYERS and N. E. STEENROD, The group of isometries of a Riemannian manifold, Ann. Math. 40 (1939), 400-416.

[19] L. Nirenberg, S. Webster and P. YANG, Local boundary regularity of holomorphic mappings, Comm. Pure Appl. Math. 33 (1980), 305-338.

[20] G. PAtrizio, On holomorphic maps between domains in $\mathbb{C}^{n}$, Ann. Scuola Norm. Sup. Pisa Cl. Sci. 13 (1986), 267-279.

[21] S. I. PINCHUK, A boundary uniqueness theorem for holomorphic functions of several complex variables, Mat. Zametki 15 (1974), 205-212.

[22] S. I. PINCHUK, On proper holomorphic mappings of strictly pseudoconvex domains, Siberian Math. J. 15 (1974), 644-649.

[23] S. I. PINCHUK, Holomorphic inequivalence of certain classes of domains in $\mathbb{C}^{n}$, Mat. Sb. (N.S.) 111(153) (1980), 67-94.

[24] S. I. PinchuK and S. V. Khasanov, Asymptotically holomorphic functions and their applications, Math. USSR-Sb. 62 (1992), 541-550.

[25] S. I. PINCHUK, The scaling method and holomorphic mappings In: "Several Complex Variables and Complex Geometry”, Part 1 (Santa Cruz, CA, 1989), 151-161, Proc. Sympos. Pure Math., 52, Part 1, Amer. Math. Soc., Providence, RI, 1991.

[26] J-P. Ros AY, Sur une caractèrisation de la boule parmi les domaines de $\mathbb{C}^{n}$ par son groupe d'automorphismes, Ann. Inst. Fourier (Grenoble), 29 (1979), 91-97.

[27] K. Verma, Boundary regularity of correspondences in $\mathbb{C}^{2}$, Math. Z. 231 (1999), 253-299.

[28] E. Vesentini, Complex geodesics and holomorphic maps, In: Symposia Mathematica", Vol. XXVI, Rome, 1980, 211-230, Academic Press, London-New York, 1982.

[29] J. P. ViguÉ, Caractérisation des automorphismes analytiques d'un domaine convexe borné, C. R. Acad. Sci. Paris Sér. I Math. 299 (1984), 101-104. 
[30] S. WebsteR, On the reflection principle in several complex variables, Proc. Amer. Math. Soc. 71 (1978), 26-28.

[31] B. Wong, Characterization of the unit ball in $\mathbb{C}^{n}$ by its automorphism group, Invent. Math. 41 (1977), 253-257.

[32] J. Y. YU, Weighted boundary limits of the generalized Kobayashi-Royden metrics on weakly pseudoconvex domains, Trans. Amer. Math. Soc. 347 (1995), 587-614.

Department of mathematics Indian Institute of Science Bangalore 560012, India harish@math.iisc.ernet.in

Department of Mathematics Indian Institute of Science Bangalore 560012, India kverma@math.iisc.ernet.in 